\title{
Biomechanical Study of Cervical Posterior Decompression
}

\author{
Akira Hashiguchi ${ }^{1}$, Tsukasa Kanchiku ${ }^{2}$, Norihiro Nishida ${ }^{2}$, Toshihiko Taguchi ${ }^{2}$ \\ ${ }^{1}$ Department of Orthopaedic Surgery, Shimonoseki City Toyoura Hospital, Shimonoseki, Japan \\ ${ }^{2}$ Department of Orthopedic Surgery, Yamaguchi University Graduate School of Medicine, Ube, Japan
}

\begin{abstract}
Study Design: Biomechanical study.
Purpose: We examined the dynamic characteristics of different operative procedures using three-dimensional finite-element models. Overview of Literature: Cervical laminoplasty is often selected for patients requiring multilevel spinal decompression. Many laminoplasty techniques have been developed and are continually being improved.

Methods: Images were extracted for the two intervertebral joints between C3 and C5 in domestic rabbits. The following models were created: preoperative, laminectomy, Z-plasty, open door laminoplasty, French open door laminoplasty, en-bloc laminoplasty, and double-door laminoplasty. The loads required for sagittal rotation of $2.5^{\circ}, 5^{\circ}$, and $7.5^{\circ}$ were measured in vitro and respectively placed at the bottom of the $C 5$ model. Displacement of the plate in the $X, Y$, and $Z$ coordinates was measured and the rotational angles of the plate were determined.

Results: The sagittal rotation angle was greater in the laminectomy model than in the preoperative model, but was almost the same in the preoperative and Z-plasty models. Compared with the preoperative model, sagittal rotation angles were smaller in the French open door, open door, en-bloc, and double-door laminoplasty models.

Conclusions: Laminectomy and various types of laminoplasty are associated with different mechanical features, such as stability and mobility, allowing for selection of the most appropriate surgical procedure for each case.
\end{abstract}

Keywords: Laminectomy; Laminoplasty; Finite element analyses

\section{Introduction}

Cervical laminoplasty, laminectomy, and posterior decompression with fusion are often selected for patients with cervical spondylotic myelopathy (CSM) and cervical ossification of the posterior longitudinal ligament (OPLL) who require multilevel spinal decompression. Many different laminoplasty techniques have been developed and continuously improved since 1970, and they all are reported to provide stable postoperative results [1-5]. Cervical laminoplasty is broadly divided into midline splitting and unilateral open door techniques. Laminoplasty has been reported as mechanically and clinically superior to laminectomy [6,7]; however, there are no major differences between these surgical procedures and the long-term results are comparable [8-13].

Some authors have reported clinical differences in postoperative intervertebral motion ranges and stability between various cervical laminoplasty procedures $[14,15]$. However, few studies have compared different lamino-

Received Sep 25, 2017; Revised Oct 16, 2017; Accepted Oct 19, 2017

Corresponding author: Norihiro Nishida

Department of Orthopedic Surgery, Yamaguchi University Graduate School of Medicine, 1-1-1 Minami-Kogushi, Ube, Yamaguchi 7558505, Japan

Tel: +81-836-22-2268, Fax: +81-836-22-2267, E-mail: nishida3@yamaguchi-u.ac.jp 
plasty methods within the same hospital center, and a consensus on the preferred procedure has not yet been reached $[16,17]$. Similarly, only one study has compared the mechanical features of different laminoplasty techniques [18].

It is difficult to compare the outcomes of each technique when these are performed under identical conditions in actual clinical practice. To our knowledge, no such investigation has been reported. To address this research gap, the present study used normal, laminectomy, and laminoplasty models developed from the same cervical spine model. Three-dimensional finite-element analyses were performed to compare the mechanical features of these surgical techniques.

\section{Materials and Methods}

\section{In vitro}

The 3rd to 5th cervical vertebrae (C3-5) of 10-monthold domestic rabbits $(n=5)$ were used in the study. The bottom half of $\mathrm{C} 2$ to the upper half of $\mathrm{C} 6$ were extracted and the following tissues were used: bones including the vertebral body, intervertebral discs, anterior longitudinal ligament, posterior longitudinal ligament, ligamenta flava, articular capsule, and interspinal ligament. Next, C2 and the upper half of $\mathrm{C} 3$ were fixed to one spinal instrument, while $\mathrm{C} 5$ and the bottom half of C6 were fixed to another spinal instrument.

Sagittal rotations of $2.5^{\circ}, 5^{\circ}$, and $7.5^{\circ}$ were applied to the spinal instrument to which $\mathrm{C} 5$ and $\mathrm{C} 6$ were fixed and the load torque for each angle was measured. The $\mathrm{C} 5$ and C6 vertebrae were dorsally and cranially fixed at $2.5^{\circ}, 5^{\circ}$, and $7.5^{\circ}$, and the torque load at each angle was measured. The load required for each angle was measured using an actuator (Yasukawa Electric Corp., Fukuoka, Japan) and a small torque converter (Kyowa Electronic Instruments Co. Ltd., Tokyo, Japan).

Computed tomography (CT) of the cervical spine was performed in domestic rabbits and the images of the two intervertebral joints between $\mathrm{C} 3$ and $\mathrm{C} 5$ were extracted. In each CT slice, points were taken at the periphery of the cortical bone and the cancellous bone. XY coordinate data were mapped with the top of the anterior margin of the vertebral body set as $(0,0)$. The $\mathrm{Z}$ coordinate was established with the lower margin of C5 set as zero. These data were entered into ANSYS (ANSYS Inc., Can-

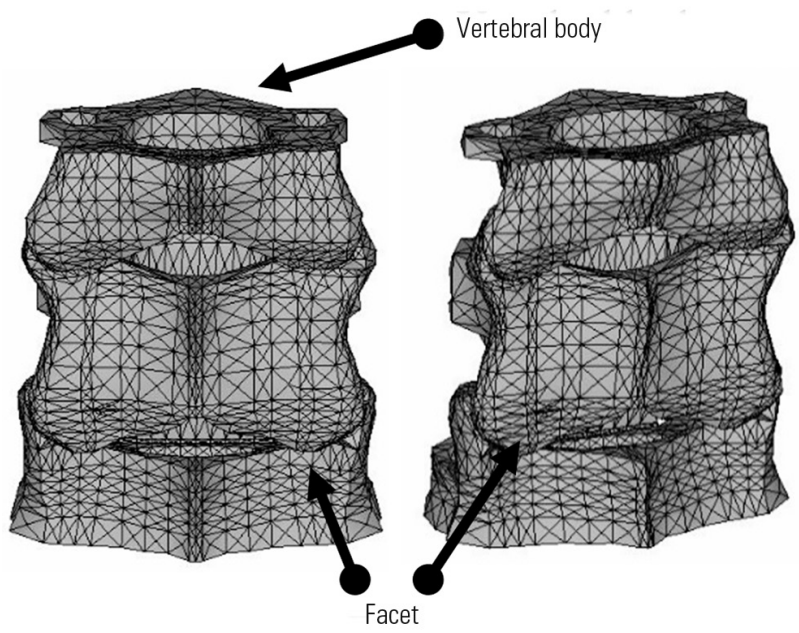

Fig. 1. Finite-element models of the section extending from the middle of $\mathrm{C} 3$ to the middle of $\mathrm{C} 5$.

onsburg, PA, USA) and various models for the section extending from middle of $\mathrm{C} 3$ to the middle of $\mathrm{C} 5$ were created (Fig. 1). The following models were created: preoperative, laminectomy, Z-plasty, open door laminoplasty, French open door laminoplasty, en-bloc laminoplasty, and double-door laminoplasty (Fig. 2).

In the Z-plasty model, the vertebral arches are thinly abraded, cut into the shape of a "Z," lifted posteriorly, and ligated. To reduce the operative time, vertebral arches on only one side were resected and opened in some vertebrae. In the French open-door laminoplasty model, the vertebral arches were vertically split, opened like French doors, and fixed with thread. In the double-door laminoplasty model, the spinous processes were vertically split and opened, and a spacer was placed between the opened spinous processes. These were then joined and fixed with the spacer in place. In the open-door laminoplasty model, the vertebral arches on one side were completely resected while those on the other side were partially resected to make them flexible before being opened and fixed. In the en-bloc laminoplasty model, a bone graft was inserted between the resected and elevated vertebral arches and the zygapophyses to keep the spinal canal expanded to an arbitrary size [1-5].

For preparation of the model, laminectomy models were first created and a vertebral arch attached to the corresponding laminectomy model. The area between the vertebral arch and the inner side of the intervertebral joint were assumed to be united and fixed in the laminoplasty models. The thickness of the hinge was set to be similar to that of the opened vertebral arches, on the assumption 
(A)

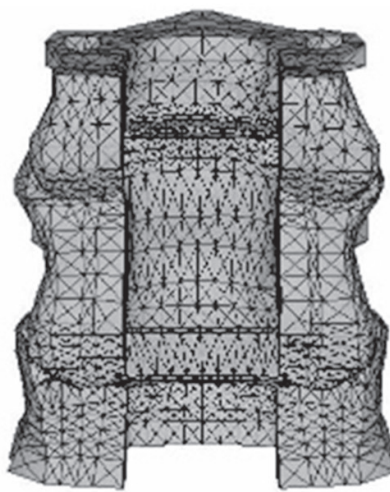

(D)

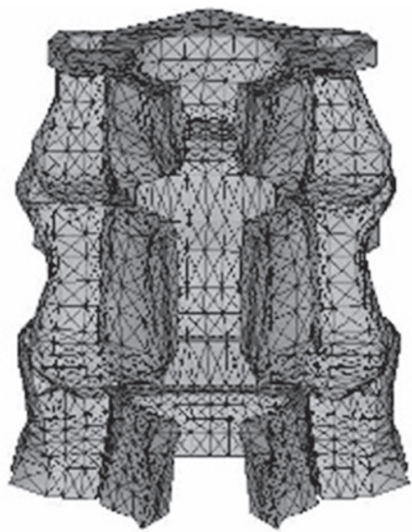

(B)
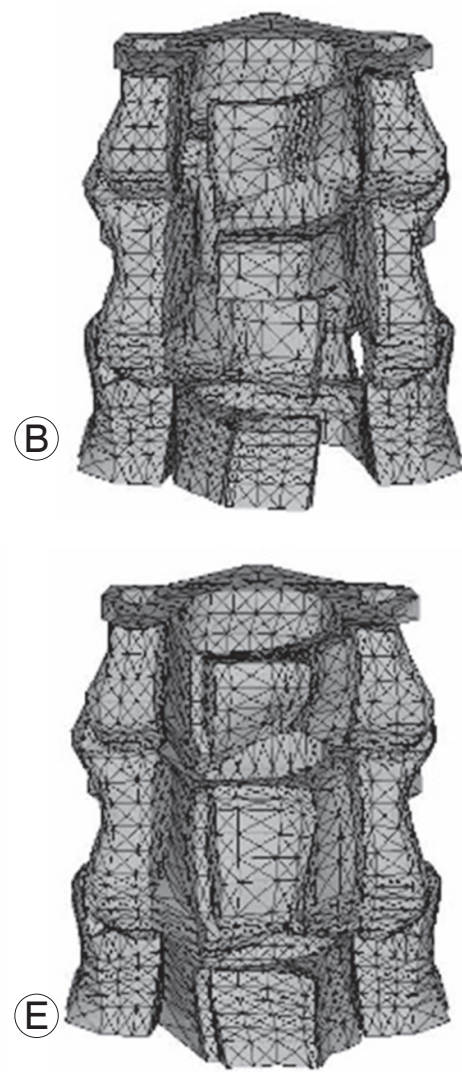

(C)
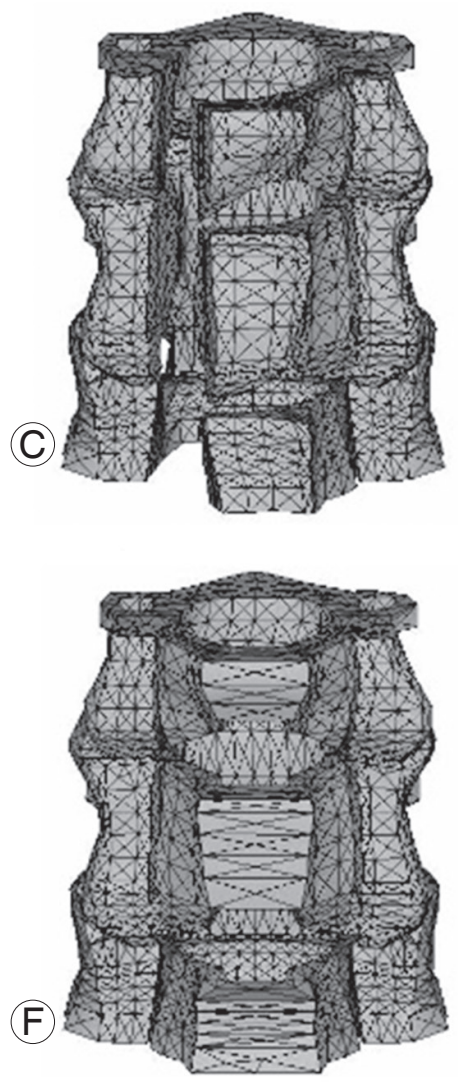

Fig. 2. The following models were created: (A) laminectomy model, (B) Z-plasty model, (C) open door laminoplasty model, (D) French open door laminoplasty models, (E) en-bloc laminoplasty model, and (F) double-door laminoplasty.

that bone union was achieved. The thickness of the vertebral arches was reduced by half in the Z-plasty model; however, the same thickness was set for all other models because these techniques did not involve abrasion of the vertebral arches.

The total number of elements in a normal mesh model was 29,925. Cortical bone, cancellous bone, end plate, annulus fibrosus matrix, nucleus pulposus, and articular cartilage were bound at eight nodes and were completely integrated. The mesh was symmetrically laid to prevent errors. The ligament was modeled as a cable element with two nodes and the intervertebral joint as a contact element. The material constant was defined using the previous literature as a reference $[19,20]$.

\section{Finite-element technique: sagittal rotation model}

Plates were placed at the upper and lower borders of the vertebral body to enable rotation of the entire plane. It was assumed that the plates and vertebral body were bound, so no effect was expected to be observed on the

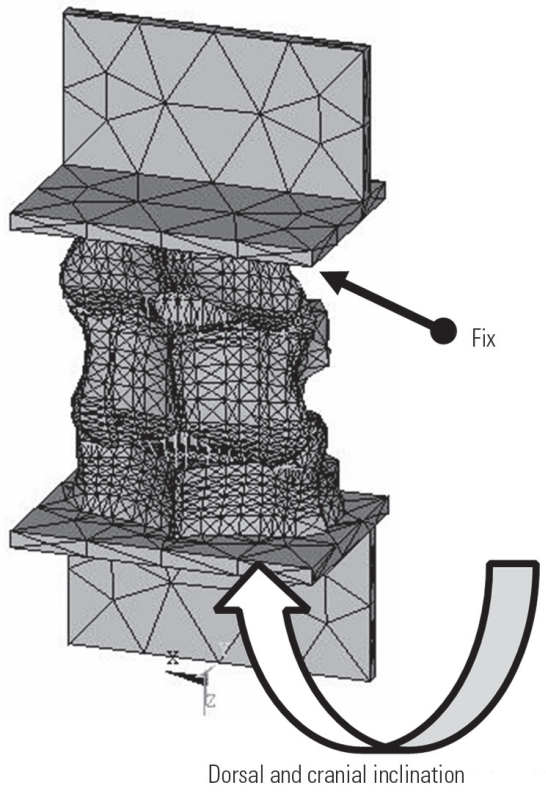

Fig. 3. Plates were placed at the upper and lower borders of the vertebral body to enable rotation of the entire plane. The loads required to dorsally and cranially rotate the cervical vertebrae by $2.5^{\circ}, 5^{\circ}$, and $7.5^{\circ}$ were measured in vitro. These loads were applied to the plate at the inferior edge. Changes in the coordinates of the plate were then measured and the rotation angles determined. 
friction coefficient of the plates with the vertebral body. Thereafter, a load was placed on the plate at the $\mathrm{C} 5$ side (Fig. 3). The loads required for sagittal rotation of $2.5^{\circ}, 5^{\circ}$, and $7.5^{\circ}$ were measured in vitro and placed on the bottom plate. The sagittal rotation of the plate in the $\mathrm{X}, \mathrm{Y}$, and $\mathrm{Z}$ coordinates was measured and rotational angles of the plate were determined.

Next, the loads required to dorsally and cranially rotate the cervical vertebrae by $2.5^{\circ}, 5^{\circ}$, and $7.5^{\circ}$ were measured in vitro. These loads were applied to the plate at the inferior edge. Changes in the coordinates of the plate were then measured and the sagittal rotation angles determined.

\section{Results}

The in vitro experiment revealed that 0.02289 Newton meter (Nm), $0.057552 \mathrm{Nm}$, and $0.099408 \mathrm{Nm}$ of torque were required to produce sagittal rotation of the plate of $2.5^{\circ}, 5^{\circ}$, and $7.5^{\circ}$, respectively.

\section{Finite-element model}

The above loads were placed on the bottom plate of the finite-element models (Fig. 4, Table 1). For the same applied load, the sagittal rotation angle was greater in the laminectomy model than in the preoperative model, but was almost the same in the preoperative and Z-plasty

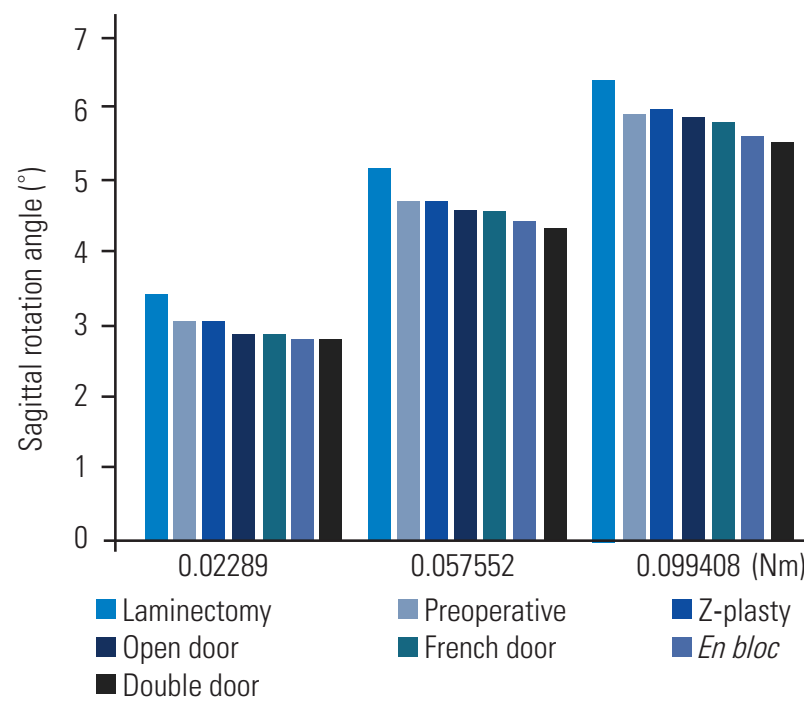

Fig. 4. The in vitro experiment revealed that 0.02289 Newton meter $(\mathrm{Nm}), 0.057552 \mathrm{Nm}$, and $0.099408 \mathrm{Nm}$ were required for sagittal rotation of the plate by $2.5^{\circ}, 5^{\circ}$, and $7.5^{\circ}$, respectively. For the same applied load, the sagittal rotation angle was larger in the laminectomy model than that in the preoperative and laminoplasty models. models. Sagittal rotation angles were smaller in both the French open door and open door laminoplasty models than that of the preoperative model. Sagittal rotation angles were smaller in the en-bloc and double-door laminoplasty models compared with that of the other laminoplasty models.

\section{Discussion}

Technical advances and stable long-term results have shown that cervical laminoplasty plays a central role in the treatment of CSM and cervical OPLL in clinical settings [9-13]. Although there are limitations when involvement of anterior compression elements is large, the procedure can be used in many patients [21-25]. Taguchi [9] reported stable results in patients after Z-plasty. Satomi et al. [10], Ogawa et al. [11], and Chiba et al. [12] have all reported stable results for open door laminoplasty; Chiba et al. [12] further reported that a brake was placed on intervertebral mobility. Seichi et al. [13] reported that mean mobility decreased from $36^{\circ}$ to $8^{\circ}$ following double-door laminoplasty. Consequently, laminoplasty is clinically considered to be a superior operative procedure and to yield good braking capacity.

A number of biomechanical studies have been published on changes in the dynamic characteristics of the spine following cervical spine surgery. Saito et al. [26] conducted a study on laminectomy using a two-dimensional finite-element model and reported that resection of the posterior ligament (i.e., ligamenta flava, supraspinous, and interspinous ligaments) shifts the load applied to the spine to the intervertebral joints where it is readily transmitted to the vertebral body. Voo et al. [27] studied intervertebral joint resection models and reported that the load imposed on the annulus increases after surgery.

Table 1. Angle changes of each surgical procedures

\begin{tabular}{lccc} 
& $0.02289 \mathrm{Nm}$ & $0.057552 \mathrm{Nm}$ & $0.099408 \mathrm{Nm}$ \\
\hline Laminectomy & 3.43 & 5.17 & 6.39 \\
\hline Preoperative & 3.01 & 4.72 & 5.94 \\
\hline Z-plasty & 2.95 & 4.74 & 6.03 \\
\hline Open door & 2.88 & 4.6 & 5.86 \\
\hline French door & 2.86 & 4.57 & 5.8 \\
\hline En bloc & 2.78 & 4.44 & 5.62 \\
\hline Double door & 2.76 & 4.37 & 5.52 \\
\hline
\end{tabular}


Based on experiments using domestic rabbits, Toh et al. [28] reported that muscles play a role as a dynamic stabilizer in maintaining alignment of the entire cervical spine. They also showed that the ligamenta flava binds the vertebral arches of adjacent segments, thus contributing as a static stabilizer for intervertebral stability. By experimenting with articular capsule resection, Zdeblick et al. [29] showed that intervertebral instability increased in $>50 \%$ of cases following joint capsule resection.

Although there have been several studies on cervical spine biomechanics, differences between surgical procedures have not been well studied. The general consensus is that laminoplasty is clinically and biomechanically superior to laminectomy for achieving intervertebral stability $[6,7,14,15,30]$. However, only a few studies have described the biomechanical results of different surgical procedures of laminoplasty [19].

To our knowledge, the present study is the first to analyze preoperative, laminectomy, and multiple laminoplasty models using the finite-element method and to verify their mechanical characteristics.

Ratliff and Cooper [14] reported that the range of motion was reduced by $46 \%$ for open door laminoplasty and $50 \%$ for double-door laminoplasty relative to preoperation. This supports our finding that the sagittal rotation angle of double-door laminoplasty is smaller than that of open door laminoplasty [14]. Taguchi [9] reported that Zplasty showed superior stability compared to laminectomy, which is also consistent with the results of our study. In addition, Hayashi et al. [17] reported that the range of motion after double-door laminoplasty was lower than that observed after open door laminoplasty. In other words, they found that the stability was higher, which further supports the results of the current study. Hayashi et al. [17] also reported that the difference in average stability of the range of motion from $\mathrm{C} 2$ to $\mathrm{C} 7$ between open door laminoplasty (from average of $38^{\circ}$ and $20^{\circ}$, difference of $18^{\circ}$ ) and double-door laminoplasty (from average of $39^{\circ}$ and $15^{\circ}$, difference of $24^{\circ}$ ) was $6^{\circ}$. In the present study, the difference in sagittal rotation angle between the open door and double-door laminoplasty models under a $0.0099408 \mathrm{Nm}$ load was $0.36^{\circ}$. We believe that the smaller angle is because of analysis of only two intervertebral segments and because rabbits have cervical vertebra smaller than that of humans. The difference in angle would likely be larger after correction for the size of the vertebral body and increasing the number of interverte- bral segments.

However, these reports are clinical studies and thus do not consider the same model. In contrast to such clinical studies, Kode et al. [31] created an intact model, a laminectomy model, and a laminoplasty model (not open door laminoplasty, but similar to en-bloc laminoplasty) using five human cadaveric specimens and analyzed differences in movement after application of $2 \mathrm{Nm}$ force. They observed a significant difference between the laminectomy and intact (preoperative) models; whoever, no significant difference between the laminoplasty and preoperative models was observed. In our study, the difference in angle between the preoperative and laminectomy models was $0.42^{\circ}$ under $0.02289 \mathrm{Nm}$ (approximately 1,000-fold lower force than in Kode's study), while the difference in angle between the preoperative and en-bloc laminoplasty models was $0.23^{\circ}$. We considered that the difference between $0.23^{\circ}$ and $0.42^{\circ}$ was significant. The preoperative model, Z-plasty, open door laminoplasty, and French door laminoplasty methods showed almost equivalent stability under $0.057552 \mathrm{Nm}$ and $0.099408 \mathrm{Nm}$, although there was a tendency for better stabilization with open door laminoplasty and French door laminoplasty compared with the preoperative model. It should be noted that enbloc laminoplasty and double-door laminoplasty may show better stabilization compared with the preoperative and Z-plasty models. There is also a tendency for better stabilization with en-bloc laminoplasty and double-door laminoplasty compared with open door laminoplasty and French door laminoplasty.

Our study has several limitations. First, only two intervertebral segments were examined. Second, the alignment of the entire cervical spine, such as the anterior and posterior curvatures, was not taken into account. Third, the reconstructed vertebral arches or implanted bone grafts were assumed to fuse with the surrounding bones, and so the possible effects of hinge thickness or drilling sites of the lateral grooves were not taken into consideration. The results were not affected by other soft tissues. Possible effects of posterior ligaments that may be preserved by some surgical procedures, such as the nuchal ligament, were not taken into consideration. In addition, age-related deterioration of bone strength and degeneration of the intervertebral discs was not considered. Furthermore, the mesh was symmetrically laid to facilitate analysis. Since the analysis was immediately conducted after surgery between two intervertebral segments, it is difficult to assess 
how this affects long-term results. And only the simulation result of the finite-element method cannot distinguish statistically significant difference.

Overall, our results suggest that the laminectomy model might be associated with more instability compared with the preoperative model. Moreover, the laminoplasty models were associated with comparable or higher stability compared with the preoperative and laminectomy models. Each laminoplasty model had characteristic mechanical features; the finite-element analyses also revealed the superiority of all laminoplasty techniques.

\section{Conclusions}

Cervical spine models of 2-level laminectomy, preoperative conditions, and various laminoplasty techniques were studied by finite-element analysis to compare their mechanical features. Laminectomy and various types of laminoplasty are associated with different mechanical features, such as stability and mobility, thus allowing selection of the appropriate surgical procedure for each case.

\section{Conflict of Interest}

No potential conflict of interest relevant to this article was reported.

\section{Acknowledgments}

Type of contribution of the authors: Akira Hashiguchi and Norihiro Nishida wrote and prepared the manuscript, and all of the authors participated in the study design. All authors have read, reviewed, and approved the article.

\section{References}

1. Kawai S, Sunago K, Doi K, Saika M, Taguchi T. Cervical laminoplasty (Hattori's method): procedure and follow-up results. Spine (Phila Pa 1976) 1988;13:1245-50.

2. Kurokawa T, Tsuyama N, Tanaka H, Kobayashi M, Machida H, Nakamura K. Double-door laminoplasty. Bessatsu Seikeigeka (Suppl Orthop Surg) 1982;2:23440.

3. Miyazaki K, Kirita Y. Extensive simultaneous multisegment laminectomy for myelopathy due to the ossification of the posterior longitudinal ligament in the cervical region. Spine (Phila Pa 1976) 1986;11:53142.

4. Hirabayashi K, Watanabe K, Wakano K, Suzuki N, Satomi K, Ishii Y. Expansive open-door laminoplasty for cervical spinal stenotic myelopathy. Spine (Phila Pa 1976) 1983;8:693-9.

5. Itoh $\mathrm{T}$, Tsuji $\mathrm{H}$. Technical improvements and results of laminoplasty for compressive myelopathy in the cervical spine. Spine (Phila Pa 1976) 1985;10:729-36.

6. Fields MJ, Hoshijima K, Feng AH, Richardson WJ, Myers BS. A biomechanical, radiologic, and clinical comparison of outcome after multilevel cervical laminectomy or laminoplasty in the rabbit. Spine (Phila Pa 1976) 2000;25:2925-31.

7. Nowinski GP, Visarius H, Nolte LP, Herkowitz HN. A biomechanical comparison of cervical laminaplasty and cervical laminectomy with progressive facetectomy. Spine (Phila Pa 1976) 1993;18:1995-2004.

8. Okada M, Minamide A, Endo T, et al. A prospective randomized study of clinical outcomes in patients with cervical compressive myelopathy treated with open-door or French-door laminoplasty. Spine (Phila Pa 1976) 2009;34:1119-26.

9. Taguchi T. Long term results of surgical procedures of cervical spondylosis myelopathy. In: Taguchi T, editor. New mook of orthopaedics. Tokyo: Kanehara Co.; 1999. p.198-204.

10. Satomi K, Nishu Y, Kohno T, Hirabayashi K. Longterm follow-up studies of open-door expansive laminoplasty for cervical stenotic myelopathy. Spine (Phila Pa 1976) 1994;19:507-10.

11. Ogawa Y, Chiba K, Matsumoto M, Nakamura M, Takaishi H, Toyama Y. Postoperative factors affecting neurological recovery after surgery for cervical spondylotic myelopathy. J Neurosurg Spine 2006;5:483-7.

12. Chiba K, Ogawa Y, Ishii K, et al. Long-term results of expansive open-door laminoplasty for cervical myelopathy: average 14-year follow-up study. Spine (Phila Pa 1976) 2006;31:2998-3005.

13. Seichi A, Takeshita K, Ohishi I, et al. Long-term results of double-door laminoplasty for cervical stenotic myelopathy. Spine (Phila Pa 1976) 2001;26:47987.

14. Ratliff JK, Cooper PR. Cervical laminoplasty: a critical review. J Neurosurg 2003;98(3 Suppl):230-8.

15. Heller JG, Raich AL, Dettori JR, Riew KD. Comparative effectiveness of different types of cervical lami- 
noplasty. Evid Based Spine Care J 2013;4:105-15.

16. Nakashima H, Kato F, Yukawa Y, et al. Comparative effectiveness of open-door laminoplasty versus French-door laminoplasty in cervical compressive myelopathy. Spine (Phila Pa 1976) 2014;39:642-7.

17. Hayashi K, Yone K, Matsunaga S, et al. A comparative study of open-door laminoplasty and Frenchwindow laminoplasty for treatment of cervical multisegmental spondylotic myelopathy. Orthop Traumatol 2002;51:312-4.

18. Puttlitz CM, Deviren V, Smith JA, et al. Biomechanics of cervical laminoplasty: kinetic studies comparing different surgical techniques, temporal effects and the degree of level involvement. Eur Spine J 2004;13:21321.

19. Goel VK, Clausen JD. Prediction of load sharing among spinal components of a C5-C6 motion segment using the finite element approach. Spine (Phila Pa 1976) 1998;23:684-91.

20. Yang $\mathrm{KH}, \mathrm{Hu} \mathrm{J}$, White NA, King AI, Chou CC, Prasad P. Development of numerical models for injury biomechanics research: a review of 50 years of publications in the Stapp Car Crash Conference. Stapp Car Crash J 2006;50:429-90.

21. Tani T, Ushida T, Ishida K, Iai H, Noguchi T, Yamamoto H. Relative safety of anterior microsurgical decompression versus laminoplasty for cervical myelopathy with a massive ossified posterior longitudinal ligament. Spine (Phila Pa 1976) 2002;27:2491-8.

22. Taniyama T, Hirai T, Yamada T, et al. Modified K-line in magnetic resonance imaging predicts insufficient decompression of cervical laminoplasty. Spine (Phila Pa 1976) 2013;38:496-501.

23. Iwasaki M, Okuda S, Miyauchi A, et al. Surgical strategy for cervical myelopathy due to ossification of the posterior longitudinal ligament: part 2: advantages of anterior decompression and fusion over laminoplasty. Spine (Phila Pa 1976) 2007;32:654-60.
24. Seichi A, Chikuda H, Kimura A, et al. Intraoperative ultrasonographic evaluation of posterior decompression via laminoplasty in patients with cervical ossification of the posterior longitudinal ligament: correlation with 2-year follow-up results. J Neurosurg Spine 2010;13:47-51.

25. Yamazaki A, Homma T, Uchiyama S, Katsumi Y, Okumura H. Morphologic limitations of posterior decompression by midsagittal splitting method for myelopathy caused by ossification of the posterior longitudinal ligament in the cervical spine. Spine (Phila Pa 1976) 1999;24:32-4.

26. Saito T, Yamamuro T, Shikata J, Oka M, Tsutsumi S. Analysis and prevention of spinal column deformity following cervical laminectomy: I. Pathogenetic analysis of postlaminectomy deformities. Spine (Phila Pa 1976) 1991;16:494-502.

27. Voo LM, Kumaresan S, Yoganandan N, Pintar FA, Cusick JF. Finite element analysis of cervical facetectomy. Spine (Phila Pa 1976) 1997;22:964-9.

28. Toh S, Kawai S, Saika M, et al. An experimental study on roles of the posterior structure of cervical spine. Orthop Traumatol 1992;40:1280-2.

29. Zdeblick TA, Abitbol JJ, Kunz DN, McCabe RP, Garfin S. Cervical stability after sequential capsule resection. Spine (Phila Pa 1976) 1993;18:2005-8.

30. Baisden J, Voo LM, Cusick JF, Pintar FA, Yoganandan N. Evaluation of cervical laminectomy and laminoplasty: a longitudinal study in the goat model. Spine (Phila Pa 1976) 1999;24:1283-8.

31. Kode S, Gandhi AA, Fredericks DC, Grosland NM, Smucker JD. Effect of multilevel open-door laminoplasty and laminectomy on flexibility of the cervical spine: an experimental investigation. Spine (Phila Pa 1976) 2012;37:E1165-70. 